\title{
LA SCAPOLINE APPLIQUÉE SUR CORPUS. L'EXEMPLE DU PRONOM $\mathrm{ON}$
}

\author{
Coco Norén
}

Armand Colin | «Langue française »

2009/4 n 164 | pages 137 à 148

ISSN 0023-8368

ISBN 782200925727

Article disponible en ligne à l'adresse :

https://www.cairn.info/revue-langue-francaise-2009-4-page-137.htm

Distribution électronique Cairn.info pour Armand Colin.

(C) Armand Colin. Tous droits réservés pour tous pays.

La reproduction ou représentation de cet article, notamment par photocopie, n'est autorisée que dans les limites des conditions générales d'utilisation du site ou, le cas échéant, des conditions générales de la licence souscrite par votre établissement. Toute autre reproduction ou représentation, en tout ou partie, sous quelque forme et de quelque manière que ce soit, est interdite sauf accord préalable et écrit de l'éditeur, en dehors des cas prévus par la législation en vigueur en France. Il est précisé que son stockage dans une base de données est également interdit. 


\section{Coco Norén}

Université d’Uppsala

\section{La ScaPoLine appliquée sur corpus. L'exemple du pronom on}

La notion de polyphonie commence à avoir une histoire dans la linguistique française. Les éléments de ce concept sont inspirés de Bakhtine, de Genette, et de Berrendonner, mais il va sans dire que ce fut Ducrot qui lui donna son nom et la concrétisa dans le Dire et le dit (1984). Depuis, le champ a été élargi et approfondi, au niveau de la théorie comme de la méthode et de l'application, et a donc été diversifié. Un des enfants de la polyphonie ducrotienne a été la ScaPoLine (2004), théorie dont l'ambition est de présenter un modèle cohérent, notamment pour ce que nous avons appelé la configuration polyphonique. Bien que nous nous soyons plus particulièrement occupés des éléments constitutifs de cette configuration, qui se place au niveau de l'énoncé, l'objet final a été d'examiner des unités plus larges et des matériaux allant au-delà des exemples canoniques.

Le résultat de cette ambition est tangible. Il y a aujourd'hui un grand nombre de travaux réalisés par différents chercheurs qui, plus ou moins fidèles à la ScaPoLine ou seulement inspirés par cette théorie, mais surtout très attachés à l'idée génératrice de la pluralité des voix, analysent la polyphonie du discours rapporté, des modes, de la négation, des connecteurs... Certaines de ces études prennent en compte un cotexte qui dépasse l'énoncé, le passage polyphonique, et traitent la notion de genre en termes polyphoniques. Il y a donc eu une ScaPoLine appliquée à divers phénomènes linguistiques, aussi bien qu'une ScaPoLine étendue au-delà de la limite de l'énoncé. Reste le reproche que l'on peut avancer à l'égard de la méthode : la ScaPoLine ne s'est que peu servie de grands corpus.

Le terme ON-polyphonique est comme tout terme conventionnel, mais on ne peut s'empêcher de le rapprocher du pronom on ${ }^{1}$. Parmi les divers emplois de

1. Pour éviter toute confusion, ON-polyphonique sera dorénavant écrit en majuscules et le pronom on en minuscules italiques. 
on, c'est la valeur d'indéfini générique qui a été reprise dans le terme - et le concept - de ON-polyphonique, la voix de tout homme. Or les emplois de on couvrent, comme on le verra, la plupart des représentations de toute la gamme des êtres discursifs dans le modèle de la ScaPoLine.

De ces brefs passages d'introduction découle le double objectif du présent article: d'une part examiner les correspondances entre les emplois du pronom on et la manifestation linguistique des êtres discursifs dans la configuration polyphonique, d'autre part montrer l'utilité des corpus afin d'approfondir le modèle théorique. Pour le lecteur impatient, nous dévoilerons déjà ici notre conclusion générale, à savoir que les aspects polyphoniques d'un discours sont un aspect important, parmi d'autres, pour caractériser un genre discursif.

\section{LES ÊTRES DISCURSIFS DE LA ScaPoLine}

Récapitulons la taxinomie des êtres discursifs de la ScaPoLine (Nølke, Fløttum et Norén $2004: 39$ ).

\begin{tabular}{|l|l|l|l|}
\hline \multicolumn{1}{|c|}{ Être discursif } & \multicolumn{1}{|c|}{ LOC } & \multicolumn{1}{c|}{ ALLOC } & \multicolumn{1}{c|}{ TIERS } \\
\hline Images & $\begin{array}{l}\text { le locuteur de l'énoncé, I0 } \\
\text { le locuteur textuel, L }\end{array}$ & $\begin{array}{l}\text { l'allocutaire de l'énoncé, a0 } \\
\text { l'allocutaire textuel, A }\end{array}$ & \\
\hline Sous-catégories & & & $\begin{array}{l}- \text { individuels } \\
- \text { collectifs } \\
\text { hétérogènes : ON } \\
\text { homogènes : L0I }\end{array}$ \\
\hline
\end{tabular}

Quelques remarques s'imposent. Il y a une certaine asymétrie apparente dans le modèle. En premier lieu nous n'avons distingué les deux images « de l'énoncé » et « textuelle » que pour le locuteur et l'allocutaire, distinction qui repose sur la dichotomie du montré et du dit. En second lieu, la diversification du tiers, issue d'une analyse plus fine de ses diverses représentations, n'a pas été effectuée pour le locuteur ou l'allocutaire. Rien n'empêche d'établir des modèles asymétriques, afin d'éviter l'esthétisme exagéré des cases, quand les données nous l'imposent. Or nous défendrons l'idée qu'il n'y a lieu ni de scinder le tiers en plusieurs instances, ni de le priver de ses deux images.

\section{LES VALEURS SÉMANTICO-RÉFÉRENTIELLES DE ON}

Avant d'aborder la question des correspondances entre les êtres discursifs et les emplois de on, nous présenterons ici les valeurs sémantico-référentielles de ce pronom et leurs équivalents référentiels approximatifs (Fløttum, Jonasson et Norén $2007: 30)$ : 


\begin{tabular}{|c|c|c|c|c|}
\hline \multicolumn{3}{|c|}{ indéfini } & \multicolumn{2}{|c|}{ personnel } \\
\hline impersonnel & générique & spécifique & neutre & stylistique \\
\hline il, ce & $\begin{array}{l}\text { tous les hommes, } \\
\text { chacun }\end{array}$ & ils, quelqu’un & nous & $\begin{array}{l}\text { je, tu, vous, il, elle, ils, } \\
\text { elles }\end{array}$ \\
\hline
\end{tabular}

Pour concrétiser ces emplois, considérons les exemples suivants (ibid.) :

Indéfini

- impersonnel : On était le 15 octobre.

- générique : On peut toujours rêver.

- spécifique: On frappe à la porte.

Personnel

- Tante, on va aller dormir maintenant

- On fait aller. On fait ce qu'on peut (pour je)

- Alors, on a bien dormi ? On est contente? (pour tu)

- On s'est encore disputés, tous les deux? (pour vous)

- Et on s'ennuie! (pour elle, à propos d'Emma dans Mme Bovary, voir XX)

- (Ils vinrent à Tostes.) On s'expliqua (pour ils)

Comme on n'a que deux traits sémantiques invariables, dont l'un est de dénoter un ou plusieurs êtres humains, et l'autre est de remplir la fonction de sujet grammatical, ce pronom peut être la représentation de n'importe quel être discursif, selon qu'il s'agit de l'emploi indéfini ou personnel, ce qui sera montré dans les sections suivantes.

\section{ON COMME LOC ET ALLOC}

Pouvant assimiler n'importe quelle référence, on peut dans ses emplois personnels stylistiques être la représentation du locuteur, dans son image de locuteur textuel ${ }^{2}$, à condition qu'on accepte le cotexte local pour l'interprétation. Voyons l'exemple (1).

(1) Homais se présenta.

- Je compte sur vous, fit le docteur. Sommes-nous prêts? En marche!

Mais l'apothicaire, en rougissant, avoua qu'il était trop sensible pour assister à une pareille opération.

- Quand on est simple spectateur, disait-il, l'imagination, vous savez, se frappe ! Et puis j'ai le système nerveux tellement... (Bovary, 256)

2. On ne peut pas représenter le locuteur de l'énoncé, puisque dans le cas où il figure dans une proposition montrée, il prend nécessairement une référence indéfinie générique, bloquant ainsi cette interprétation du locuteur. Voir infra. 
Dans l'énoncé en forme de discours direct, "Quand on est simple spectateur, disait-il, l'imagination, vous savez, se frappe!», la référence de on est sous-déterminée et pointe vers une interprétation indéfinie générique. Or, d'une part le contexte suggère que on réfère au locuteur Homais, vu qu'un " apothicaire » n'effectue pas d'opération et ne peut donc être que spectateur, et d'autre part qu'une coréférentialité s'établit entre on et je dans l'énoncé suivant «j'ai le système nerveux tellement... ».

Cette même flexibilité sémantique permet à on de jouer le rôle d'allocutaire textuel, ce qui ressort de l'exemple (2) :

(2) Il [Bovary] voulut emmener Canivet dans la pièce voisine. Charles le suivit. —Elle est bien mal, n'est-ce pas ? Si l'on posait des sinapismes ? je ne sais quoi! Trouvez donc quelque chose, vous qui en avez tant sauvé !

Charles lui entourait le corps de ses deux bras, et il le contemplait d'une manière effarée, suppliante, à demi pâmé contre sa poitrine.

(Bovary, 414)

On prend ici la valeur personnelle stylistique de "vous", ce qui ressort de l'énoncé «Trouvez donc quelque chose, vous qui en avez tant sauvé ! » où il y a une coréférentialité qui s'instaure entre on, le sujet de la forme impérative trouvez et le pronom vous.

Ainsi, nous pouvons constater que on peut représenter le locuteur et l'allocutaire, mais ce n'est pas là notre propos, étant donné que le fil rouge de cet article est de dégager la relation entre les emplois du pronom on et les instances énonciatives des tiers, notamment le ON-polyphonique.

\section{SCISSION DES TIERS MISE EN CAUSE}

L'analyse des protagonistes de l'énonciation, locuteur et allocutaire, a été faite de façon extensive dans les travaux antérieurs, tandis que les tiers ont été quasiment abandonnés à leur sort, en dépit de leur sous-catégorisation dans le modèle, plus fine que celle des autres êtres discursifs. Revoyons l'argumentation que nous avons avancée en 2004. Nous avons postulé que "Les tiers sont les êtres discursifs qui peuvent être représentés par les pronoms de la troisième personne, par les noms propres ou par les syntagmes nominaux ayant une référence non générique » (Nølke, Fløttum et Norén 2004 : 38sq) sans élaborer la nature des tiers individuels. Rétrospectivement, l'absence d'une telle description apparaît comme un manque.

La distinction entre individuel et collectif répond à priori à notre intuition, comme nous l'avons avancé : « tout porte à croire qu'il faille distinguer les tiers individuels des tiers collectifs que nous symboliserons par $\mathrm{ON} »$ (ibid: $38 \mathrm{sq}$ ). Cependant, une fois que l'on est confronté à des exemples authentiques, cette distinction ne va pas de soi. Certaines occurrences de on ne posent aucun problème, comme dans l'exemple (3) : 
(3) Il [Homais] conta des histoires. Charles se surprit à rire ; mais le souvenir de sa femme, lui revenant tout à coup, l'assombrit. On apporta le café ; il n'y pensa plus. (Bovary, 69)

Il s'agit bien ici d'un tiers individuel, puisque selon la conception générale du monde, le café est servi par une seule personne. Le contexte de "servir du café » aussi bien que le passé simple indiquent l'individualité de on, donc ici à valeur indéfinie spécifique, puisque la personne en question n'a pas été introduite auparavant et que son identité ne joue aucun rôle important dans le texte.

Lorsque la référence de on est indéfinie générique, il s'agit d'une collectivité dont l'identité n'est pas connue :

(4) Ses cheveux châtains tombaient dessus, plats et bien peignés. Elle remarqua ses ongles, qui étaient plus longs qu'on ne les portait à Yonville. C'était une des grandes occupations du clerc que de les entretenir ; et il gardait, à cet usage, un canif tout particulier dans son écritoire. (Bovary, 97)

Bien que la référence de on soit limitée par le complément circonstanciel à Yonville, il s'agit d'une collectivité dont les membres sont inconnus, et la prédication ne renvoie pas à un événement spécifique. Il s'agit donc sans aucun doute d'une collectivité à contours flous. Mais que faire des cas qui se placent entre ces deux extrêmes facilement discernables, notamment ceux où on a une valeur personnelle stylistique, correspondant à ils ou elles ?

(5) Il [Rodolphe] la [Emma] revit le soir, pendant le feu d'artifice ; mais elle était avec son mari, madame Homais et le pharmacien, lequel se tourmentait beaucoup sur le danger des fusées perdues; et, à chaque moment, il quittait la compagnie pour aller faire à Binet des recommandations. [...]

$[x x x]$

Et, s'étant salués, on se tourna le dos. (Bovary, 121-122)

L'identité des personnes incluses dans la collectivité est connue. Les cinq personnes auxquelles on fait référence sont Rodolphe (il) et Emma (la), Charles (son mari), et Madame et Monsieur Homais (le pharmacien). Par conséquent, il est question de tiers, individuels parce que leur nombre et leur identité sont connus, collectifs, parce qu'ils sont plusieurs. On est sans aucun doute une représentation de l'être discursif tiers, mais partage les caractéristiques et du tiers individuel, puisqu'il n'y a pas de contours flous, et du tiers collectif, puisque les référents sont plusieurs et qu'ils peuvent prendre la parole, comme on le voit dans le passage cité.

La conclusion est claire: si, pour certains phénomènes linguistiques, il semble pertinent de faire la distinction entre la catégorie des tiers individuels et celle des tiers collectifs, cette dichotomie ne peut rendre compte des divers emplois de on. C'est pourquoi nous proposons de garder dans le modèle de base de la ScaPoLine une catégorie plus générale des tiers, sans pousser la modélisation à un niveau inférieur. 


\section{TIERS HÉTÉROGÈNE OU HOMOGÈNE}

Selon la ScaPoLine, l'échelle entre les tiers collectifs hétérogènes (ONpolyphonique) et collectifs homogènes (LOI) repose sur deux paramètres (ibid : 39) : d'une part la diversité ou l'unicité des voix à l'intérieur du collectif, d'autre part leurs représentations linguistiques. ON serait composé d'un nombre de membres distinguables, susceptibles de prendre la parole, et pouvant inclure ou non d'autres locuteurs virtuels, y compris L et A. Il peut être représenté par le pronom on à référence indéfinie lorsque celui-ci est accompagné d'un verbe qui instaure un lien énonciatif de responsabilité, comme les verbes de parole, de croyance ou d'opinion. La genèse de cette notion découle d'une analyse des expressions du type on dit que pour élucider leurs emplois dans les argumentations d'autorité. Un exemple type serait :

(6) Des aboiements continus se traînaient au loin, quelque part.

- Entendez-vous un chien qui hurle? dit le pharmacien.

- On prétend qu'ils sentent les morts, répondit l'ecclésiastique. C'est comme les abeilles; elles s'envolent de la ruche au décès des personnes. (Bovary, 427)

Selon la définition posée en 2004, on dans l'exemple ci-dessous est également une représentation des tiers collectifs, puisqu'il en remplit toutes les conditions nécessaires.

(7) Ce fut un vacarme qui s'élança d'un bond, monta en crescendo, avec des éclats de voix aigus (on hurlait, on aboyait, on trépignait, on répétait : Charbovari! Charbovari!), puis qui roula en notes isolées, se calmant à grand-peine, [...] (Bovary, 49)

La scène est connue : c'est le premier jour d'école de Charles. Il rentre en classe, le professeur le présente à ses futurs camarades, qui se mettent à vociférer son nom. L'ensemble d'individus représentés par on est certes limité - il y a un nombre restreint d'écoliers dans chaque classe - mais collectif - ils sont plusieurs. Leur identité spécifique est inconnue, mais ils peuvent apparemment prendre la parole puisqu'ils hurlent et répètent, et même aboient. Pourtant, il ne semble pas pertinent d'identifier ce on comme ON-polyphonique, la description des activités des écoliers ne contribuant pas au caractère polyphonique du texte.

Finalement, en 2004, nous avons posé que la LOI est une collectivité à contours flous, indivisible, équivalant à la doxa, les idées reçues, les vérités éternelles. Lorsque la LOI est la source d'un point de vue, celle-ci n'est pas explicitée. Ce n'est que dans les présuppositions que la LOI peut intervenir. La différence entre l'exemple (6) ci-dessus avec on prétend et l'expression il paraît que n'est pas dans la configuration polyphonique; dans les deux cas il s'agit d'un tiers responsable d'un point de vue. La différence réside dans le fait que dans le premier cas le tiers est représenté linguistiquement, contrairement à ce qui est le cas avec il paraît que. 


\section{TIERS DE L’ÉNONCÉ ET TIERS TEXTUEL}

Dans Nølke, Fløttum et Norén (2004: 38) la distinction entre véridiction et monstration a servi d'instrument pour distinguer, en ce qui concerne le locuteur et l'allocutaire, les formes représentant les images de l'énoncé de celles des images textuelles. Nous rappelons que le modus, résultat de la monstration, ne peut pas faire l'objet d'une question, d'une véridiction ou d'une négation. Nous n'avons pas établi de distinction entre deux images des tiers, sans pour autant exclure la possibilité d'en voir une :

Nous n'avons pas trouvé de preuve empirique selon laquelle il conviendrait d'établir une distinction entre les tiers de l'énoncé et les tiers textuels. La pertinence de cette sous-catégorisation éventuelle, parallèle à celle établie pour le locuteur et l'allocutaire, reste donc une question ouverte. (Nølke, Fløttum et Norén $2004: 38$ )

Cette preuve empirique est fournie par des occurrences de on, qui s'insère aussi bien dans la partie assertée d'une proposition que dans une proposition parenthétique montrée. Voyons les exemples (8) et (9) ci-dessous :

(8) Le portrait de Rodolphe lui sauta en plein visage, au milieu des billets doux bouleversés. On s'étonna de son découragement. (Bovary, 444)

(9) Puis, dès qu'il fut parti, M. Homais dit au médecin :

- [...] Dépêchez-vous! Lagardy ne donnera qu'une seule représentation ; il est engagé en Angleterre à des appointements considérables. C'est, à ce qu'on assure, un fameux lapin! il roule sur l'or ! Il mène avec lui trois maîtresses et son cuisinier! (Bovary, 298)

En faisant suivre l'énoncé par une réfutation, nous constatons que dans $\left(8^{\prime}\right)$ la négation porte sur la prédication contenant on, ce qui n'est pas le cas pour $\left(9^{\prime}\right)$ :

$\left(8^{\prime}\right) \quad$ On s'étonna de son découragement.

- Non, ce n'est pas vrai!

$\left(9^{\prime}\right) \quad C^{\prime}$ est, à ce qu'on assure, un fameux lapin !

- Non ce n'est pas vrai!

Si l'on admet que la monstration/véridiction est le critère distinctif entre les deux images des êtres discursifs, il faut également admettre que les tiers en ont deux, tout comme les autres.

Pour résumer cette première partie, voilà un tableau synoptique des correspondances que nous avons pu dégager entre les emplois de on et les êtres discursifs :

\begin{tabular}{|l|l|l|l|l|}
\hline \multicolumn{3}{|c|}{ indéfini } & \multicolumn{2}{c|}{ personnel } \\
\hline impersonnel & générique & spécifique & neutre & stylistique \\
\hline il, ce & $\begin{array}{l}\text { tous les hommes, } \\
\text { chacun }\end{array}$ & ils, quelqu'un & nous & $\begin{array}{l}\text { je, tu, vous, il, elle, ils, } \\
\text { elles }\end{array}$ \\
\hline & $\begin{array}{l}\text { ON de l'énoncé } \\
- \text { textuel }\end{array}$ & Tiers & Loc + alloc/tiers & $\begin{array}{l}\text { Loc } \\
\text { Alloc } \\
\text { Tiers }\end{array}$ \\
\hline
\end{tabular}




\section{CONFIGURATIONS POLYPHONIQUES ET GENRES EN CONTRASTE}

En appliquant la ScaPoLine sur de grands corpus, il nous est possible de réaliser des analyses comparatives, par exemple entre différents genres discursifs. Déjà en 2004, Fløttum (Nølke, Fløttum et Norén 2004) avait présenté une analyse de trois œuvres littéraires à partir de leurs caractéristiques polyphoniques $^{3}$. Reprenant l'idée de Bakhtine de la hiérarchisation des voix en la transposant dans le conceptuel de la ScaPoLine, elle a montré que le caractère polyphonique de divers textes est un trait saillant. De même, l'emploi du pronom on est fortement lié au genre discursif dans lequel il se présente (Fløttum, Jonasson et Norén 2007 : 33). À titre d'exemple, il va sans dire que l'emploi personnel neutre, correspondant à « nous », domine surtout à l'oral ou dans les dialogues écrits représentant le parlée .

Ces résultats, dans leurs prolongements, pointent l'un comme l'autre vers la conclusion que l'analyse de l'aspect polyphonique est un outil efficace dans la description d'un genre discursif. Or, genre et polyphonie ne sont pas une histoire d'œuf et de poule. Les configurations polyphoniques des énoncés qui forment un texte et les caractéristiques de plusieurs passages polyphoniques ne constitueront jamais le caractère essentiel d'un genre. Quelles que soient la théorie et la terminologie adoptées, les études sur le genre distingueront entre facteurs extra- et intra-linguistiques. Le genre est, pour nous, déterminé par les facteurs extralinguistiques tels que les participants, la localité, le but, etc. et les traits intralinguistiques, dont la polyphonie, découlent de ceux-là.

Les occurrences de on relevées dans Madame Bovary serviront de contraste à celles de notre corpus C-ParlEur, Corpus de discours du PARLement EURopéen, qui contient 919 débats tenus par les 78 députés français du Parlement Européen pendant la période avril 2006-mars 2008.

\section{TENDANCES DIVERGENTES}

Étant donné l'extrême flexibilité référentielle de on, son interprétation est fortement dépendante du contexte et ce n'est qu'une fois que l'établissement du référent est mis en place que l'analyse polyphonique peut se faire. Certains indices, et dans certains cas rares, des critères peuvent être discernés. Il s'agit de syntagmes nominaux disloqués ou de pronoms qui sont coréférentiels à on, d'accords du participe passé et de compléments circonstanciels qui donnent une valeur spécifique au pronom. D'autres indices, moins tranchants, sont le temps et le type du verbe dont on est sujet, ainsi que le type de proposition

3. Fløttum constate que Bovary est caractérisé par une polyphonie mêlée, alors que Le Temps retrouvé se distingue par sa poly-hétérophonie et Le mannequin par la poly-autophonie (voir Nølke, Fløttum, Norén 2004).

4. Les genres mis en contraste dans Fløttum, Jonasson et Norén 2007 sont le discours académique, la littérature réaliste du XIX ${ }^{\mathrm{e}}$ siècle et le français parlé informel. 
dans laquelle le pronom s'insère. Un seul indice est rarement suffisant : l'interprétation finale dépend donc d'un faisceau d'indices.

Bien que les données quantitatives ne puissent pas rendre compte, et encore moins expliquer, la nature intrinsèque des genres, elles peuvent servir à mettre en évidence, au niveau structurel, les différences dans l'emploi de on. Pour avoir une indication quantitative de la répartition des temps, nous avons comparé les 353 occurrences de on dans Bovary avec 353 occurrences de C-ParlEur (sur les 860 en tout). Rien ne laisse penser que ces 353 on, relevés au hasard dans les débats avec le logiciel de concordance Wordsmith-Tools, ne seraient pas représentatifs de l'ensemble. Or, il est évident que Bovary n'est pas un corpus compris comme représentatif d'un genre littéraire. Nous espérons cependant que cette méthode montre qu'il est fructueux de comparer des ensembles de données de natures différentes. Il s'agit non seulement de trouver les divergences polyphoniques entre les genres, mais également de dégager les possibilités de configurations polyphoniques communes, qui appartiennent au système de la langue.

Un des indices évoqués pour l'interprétation de on est celui du temps du verbe qui l'accompagne. Lorsque le pronom on est le sujet d'un verbe à un temps générique, itératif ou non factuel comme le présent ou l'imparfait, il est plus susceptible d'avoir une interprétation générique, et d'être donc une réalisation linguistique du tiers ON-polyphonique. Voici le résultat de ce décompte :

\begin{tabular}{|l|c|c|c|c|c|c|c|c|}
\hline & présent & p.c. & imp & p.s. & p.q.p & fut & fut. péri. & subj \\
\hline Bovary & 127 & 15 & 249 & 93 & 30 & 4 & 3 & 40 \\
\hline C-ParlEur & 256 & 26 & 15 & 0 & 2 & 30 & 4 & 5 \\
\hline
\end{tabular}

Trois explications, non exclusives, sont possibles pour expliquer la forte prédominance du présent dans C-ParlEur par rapport à Bovary. La première est banale ; il s'agit, en ce qui concerne Bovary, d'une narration fictive dans le passé alors que les débats montrent un discours argumentatif ayant le présent comme point de référence. Cela découle automatiquement de la nature des genres, où l'un est raconté à l'écrit et l'autre est tenu à l'oral. Il en découle donc également une question de code, ce qui est la deuxième explication possible. La troisième, qui interagit avec les deux premières, est que les objectifs des textes divergent. Si dans l'un l'auteur veut raconter une histoire dans une esthétique qui lui est propre, on vise dans l'autre à argumenter, ce qui ne peut se faire qu'en prenant compte de l'auditoire de façon très pragmatique. Il est alors plus prudent d'évoquer les tiers à contours flous que de désigner de façon provocante un nombre d'individus bien précis. Il semble que le discours parlementaire, pour des questions de politesse, préfère des adresses indirectes où on à valeur indéfinie, générique ou spécifique s'utilise fréquemment. Le seul fait d'avoir un présent n'est évidemment pas suffisant pour affirmer que on s'utilise de préférence de façon générique dans le discours parlementaire. Cependant, en croisant cet indice avec d'autres, une image plus fiable apparaît. 
Voilà pourquoi nous examinons la nature du verbe. Les verbes modaux s'utilisent souvent avec on à référence indéfinie générique (Fløttum, Jonasson et Norén $2007: 41)$. Voyons la répartition des occurrences de on avec pouvoir ${ }^{5}$ dans Bovary (total 18) et C-ParlEur (total 47).

\begin{tabular}{|l|c|c|c|c|c|c|c|}
\hline & peut $^{\mathbf{b}}$ & ne peut & pourra & a pu & pouvait & pourrait & put \\
\hline Bovary & 5 & $\mathrm{I}$ & 0 & 0 & 4 & 2 & $\mathrm{I}$ \\
\hline C-ParlEur & 35 & $\mathrm{I}$ & 5 & $\mathrm{I}$ & 3 & 2 & 0 \\
\hline
\end{tabular}

Outre la différence du total d'occurrences de on avec pouvoir, on voit que la grande majorité se trouve au présent tandis que la distribution est plus ou moins la même pour les autres temps.

On figure avec un nombre élevé de verbes d'opinion ou de parole, ce qui est aussi un indice de l'interprétation indéfinie. En outre, une des représentations canoniques des tiers collectifs est le pronom on à référence indéfinie combiné avec un verbe qui instaure un lien énonciatif de responsabilité comme les verbes de parole, de croyance ou d'opinion. Le résultat du dépouillement du corpus donne les chiffres suivants :

\begin{tabular}{|l|c|c|c|c|c|c|c|}
\hline & dire & croire & savoir & voir & parler & appeler & comprendre \\
\hline Bovary & 15 & 3 & 15 & 120 & 4 & 3 & $\mathrm{I}$ \\
\hline C-ParlEur & 10 & $\mathrm{II}$ & 5 & $\mathrm{II}$ & 8 & 5 & 4 \\
\hline
\end{tabular}

Ces résultats semblent nier l'idée que on à valeur indéfinie est plus fréquent dans les débats. Pour les verbes dire, savoir et voir, le nombre est plus élevé dans Bovary que dans C-ParlEur. Cependant, en analysant les occurrences, nous voyons que dire et voir s'emploient en majorité dans le texte romanesque dans leur sens concret. Des tiers individuels ou des tiers collectifs déjà introduits dans le texte prennent la parole sous forme de discours rapporté direct ou indirect, ou voient une scène se dérouler. Dans C-ParlEur, dire et voir se rangent parmi les verbes cognitifs, équivalents à «penser» ou à « constater». En ce qui concerne la faible quantité de savoir dans les débats, nous y voyons la même prudence de politesse évoquée supra.

Un autre indice qui contribue à l'interprétation de on est le type de proposition dans laquelle il se présente. On générique apparaît plus souvent dans des propositions comparatives, étant donné le sens générique de celles-ci, comme dans (10) :

5. Voilà, pour la comparaison, les mêmes chiffres pour vouloir et devoir. Le nombre total pour vouloir est de 13 occurrences dans Bovary (veut 4, ne veut 1, voulait 2, voudrait 4 ), aussi bien que dans C-ParlEur (veut 7, ne veut 1, voulait 3, voudrait 2). Pour devoir: Bovary total 9 (ne doit pas 1, devait 4,

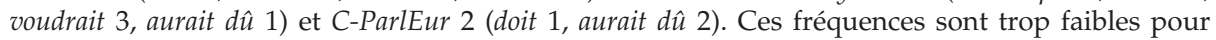
pouvoir en tirer une conclusion.

6. Il est intéressant de constater que dans C-ParlEur il y a un taux étonnamment élevé de peut avec négation : 17 peut et 18 ne peut, alors que pour Bovary il y a 4 peut et 1 ne peut. Cela mériterait une réflexion plus approfondie. 
(10) Il n'y a finalement que deux ou trois choses qui semblent intouchables et, au Conseil européen, nul n'a du reste songé à y toucher [xxx] Il s'agit de lignes rouges, comme on dit en anglais, qu'il ne faut pas franchir selon les milieux dirigeants de l’Union. (WurtzIP071023)

En comparant Bovary et C-ParlEur, nous obtenons respectivement deux et dix occurrences de on dans une comparative avec dire; comme on dit. Cependant, les deux cas relevés dans Bovary se trouvent dans des répliques, ce qui pourrait signaler que cette expression appartient plutôt à la langue parlée. Or, cela est insuffisant pour marquer une différence entre le genre littéraire et le genre politique.

Dans C-ParlEur, il est également plus fréquent de retrouver on dans une incise à caractère d'expression figée, par exemple on le sait, on le comprend, on le voit, dit-on, à ce qu'on prétend, dirait-on. Dans Bovary, il y a une seule occurrence d'incises de ce type: à ce qu'on assure (voir exemple (9)). À part le fait de répondre à plusieurs des indices de on générique, les incises sont montrées et non pas assertées. En ce sens, elles représentent l'image de l'énoncé des tiers.

Si cette investigation est partie de l'hypothèse sous-jacente que le débat parlementaire, tel qu'il apparaît dans notre corpus, est dominé par les emplois de on indéfini générique ou spécifique, et par conséquent des représentations du tiers collectif, qui peut ou non être du type ON-polyphonique, nous avons dû remettre en question sa validité. Bien que cette hypothèse réponde à notre intuition, aucun des indices interprétatifs de on n'a soutenu cette idée de façon univoque.

\section{EN GUISE DE CONCLUSION}

Dans cet article nous avons voulu illustrer une méthode descriptive des traits polyphoniques particuliers à différents genres en prenant l'exemple du pronom on et de ses emplois dans deux types de textes de caractères différents. L'étude se base d'un côté sur des données tirées de Bovary, qui a déjà été l'objet de multiples travaux sur la polyphonie. Ce texte décrit un univers fictif, dans la tradition narrative du roman réaliste du XIX ${ }^{\mathrm{e}}$ siècle. De l'autre côté, nous avons dépouillé les débats français au Parlement Européen, qui n’ont rien de fictif et qui sont essentiellement argumentatifs, intra-linguistiquement aussi bien qu'extra-linguistiquement parlant.

La comparaison a donné plusieurs résultats : si dans Bovary le pronom est le plus souvent accompagné d'un verbe à l'imparfait ou au passé simple, les débats se placent dans une sphère temporelle du maintenant, faisant parfois référence au passé avec le passé composé ou au futur avec le futur simple ou le futur périphrastique. Ceci découle principalement du fait que ces deux textes ont des points de référence temporels divergents, ce qui ne touche pas aux aspects polyphoniques proprement dits. Cependant, ce résultat, lié au fait que on dans les débats se combine avec un plus grand nombre de verbes d'opinion, de cognition ou de parole suggère l'idée que on à référence indéfinie générique est plus fréquent dans ce genre. 
La deuxième observation est que C-ParlEur offre plus de types et d'occurrences d'incises et de propositions comparatives que Bovary. Nous y voyons deux explications possibles, les deux reposant sur le genre. D'une part le caractère argumentatif de C-ParlEur peut expliquer le fait que le locuteur, sujet parlant, ait pour but de faire adhérer les auditeurs à l'argument avancé. Ce but se reflète dans la configuration linguistique polyphonique où le locuteur montre l'image d'un tiers responsable d'un point de vue, ce tiers incluant par défaut l'allocutaire. D'autre part, les incises ayant on comme sujet semblent appartenir à la langue parlée, étant donné que toutes les occurrences relevées dans Bovary figurent dans les répliques.

Finalement, il semble que l'emploi de on dans les débats peut être une stratégie de prudence qui autorise à critiquer un point de vue ou une action sans pour autant avoir à identifier le responsable, ce qui évite les actes menaçants. $C^{\prime}$ est pourquoi nous obtenons une fréquence assez élevée de cas avec un pronom objet à la première personne, comme dans on nous a dit, ce qui exclut le locuteur de la référence de on.

Pour arriver à ces résultats, nous avons dû en premier lieu nous focaliser sur les définitions et catégorisations antérieures des êtres discursifs tiers et montrer les problèmes que ceux-là posent pour l'analyse des extraits authentiques. Cela nous met devant une bifurcation théorique. Ou bien on met en place une catégorisation plus fine des êtres discursifs textuels, prenant en compte des cas d'exclusion et d'inclusion du je et du $t u$, du nombre d'individus concernés, de leur possibilité de prendre la parole ou non..., l'hétérogénéité des cas relevés risquant de nous faire aboutir à une multitude de catégories peut-être incontrôlable, ou bien on généralise les catégories à même d'assumer une gamme plus large d'emplois, en gardant la trichotomie de base : locuteur, allocutaire et tiers, qui est, comme le lecteur a pu le comprendre, la solution ici proposée.

Il nous a semblé pertinent de mettre en place une distinction entre l'image de l'énoncé et l'image textuelle des tiers, dans la logique déjà appliquée pour le locuteur et l'allocutaire.

Nous revenons donc à notre conclusion générale déjà exposée en introduction. Les aspects polyphoniques d'un discours sont un aspect important, parmi d'autres, pour caractériser un genre discursif, et pour les dégager il est indispensable de prendre en compte des ensembles de données plus larges. On n'est donc qu'au tout début d'un long cheminement.

\section{Références}

DUCROT, O., 1984, Le dire et le dit, Paris: Minuit.

FLØTTUM, K., JONASSON, K., \& NORÉN, C., 2007, On - pronom à facettes, Bruxelles : Duculot-De Boeck. NøLKE, H., FLøTtUM, K., \& NORÉN, C., 2004, ScaPoLine. La théorie scandinave de la polyphonie linguistique,

Paris : Kimé. 